\title{
ATTENUATION OF SHEAR WAVES IN THE UPPER AND LOWER MANTLE
}

\author{
By Robert L. Kovach and Don L. Anderson
}

\begin{abstract}
The attenuation of seismic waves is a direct measure of the absorption due to nonelastic processes in the earth. The well known difficulties in obtaining body wave amplitude decrement data have been avoided by studying the spectral ratios of multiple $S c S$ and $s S c S$ phases from two deep focus earthquakes recorded at near normal incidence. The average $Q$, for shear, in the mantle is about 600 for the frequency range 0.015 to $0.07 \mathrm{cps}$. Assuming that equal radiation occurs upwards and downwards from the source the average $Q$ for the upper $600 \mathrm{~km}$ of the mantle is determined to be about 200 and about 2200 for the rest of the mantle. The value for $Q$ at the base of the mantle is at least 5000 for shear waves.
\end{abstract}

\section{INTRODUCTION}

The attenuation of seismic energy in the mantle is an important parameter in discussions of source mechanism, earthquake magnitude and the range of detectability of seismic signals. Attenuation, being a manifestation of anelasticity, also has a direct bearing on discussions of the phase, composition and temperature of the earth's mantle. The main limitation in obtaining meaningful seismic wave attenuation data is that measurements are restricted to the surface of the earth. The classical method is to measure and correlate amplitudes from ray to ray along the earth's surface utilizing information from many earthquakes and recording stations and attempting to infer the spatial decay rate of a pulse along a given ray. It is well known that the differences between events, instruments and recording sites tend to obscure the amplitude differences of the seismic phases.

There are three possible ways to completely remove the effects of source and instrument. One method is to measure the rate of decay of successive transits of surface waves past a single station. Since these waves are guided by the surface of the earth we can obtain the spatial decay rate along the path. Another method is to determine the time rate of decay of peaks associated with the free oscillations of the earth. The complications due to the source are replaced by complexities of interpretation which, however, are now well in hand (Anderson and Archambeau, 1964). The third method is to consider seismic rays which have repeatedly traversed the same path and to compare amplitudes after successive passages. One such ray is the shear wave which travels between the surface and the core of the earth at near normal incidence. By studying spectral ratios of successive $S c S$ and $s S c S$ phases at near normal incidence we are able to remove the effect of the source and extract meaningful amplitude decrement data over a fairly wide frequency range. This is the method used in this paper.

Attenuation effects for propagating waves can be represented by a factor exp $(-k D)$ where $k$ is the coefficient of absorption and $D$ is distance along the ray. For a propagating monochromatic shear wave $k=\pi / \beta T Q$ where $\beta$ is the shear velocity, $T$ is the period and $Q^{-1}$ is the specific absorption coefficient of the material. 
There are several published estimates of the average $Q$ of the mantle for shear waves. Press (1956) studied the amplitude decrement of shear waves reflected from the earth's core at near normal incidence but excessive reverberations and the low sensitivity of the seismographs used limited the amount of useful data. A value of 500 was obtained for the dimensionless quality factor $Q$ for the whole mantle for 11 second shear waves. Gutenberg (1958) determined a $Q$ of 700 for 12 second shear waves and a $Q$ of 400 for 24 second shear waves but his measurements were severely limited by corrections for source, instrument and differences in path.

Otsuka (1963) interpreted the spectra of $S c S$ from two deep focus earthquakes with the assumption that the source was an impulse composed of equal amplitudes for all frequencies. With this assumption the slope of the spectrum, corrected for instrumental response, yields the absorption coefficient. Otsuka assumed a solid friction mechanism and estimated an average $Q$ of at least 340 for shear waves in the mantle. This method does not require an absolute measurement of amplitude but the assumption regarding the source is a severe limitation. A similar method was

TABLE 1

Earthquakes and Stations Selected for Study

\begin{tabular}{|c|c|c|c|c|c|}
\hline Date & Origin Time & Location & Station & $\Delta^{\circ}$ & $\begin{array}{l}\text { Wave } \\
\text { Type }\end{array}$ \\
\hline Sept. 29,1962 & $15 \mathrm{~h} 17 \mathrm{~m} 47.7 \mathrm{~s}$ & $27.0 \mathrm{~S} 63.6 \mathrm{~W}$ & LaPaz, Bolivia & 11.25 & $S H$ \\
\hline \multirow{4}{*}{ Dec. 8,1962} & $21 \mathrm{~h} 27 \mathrm{~m} \mathrm{18s}$ & $25.8 \mathrm{~S} 63.4 \mathrm{~W}$ & Antofagasta, Chile & 6.7 & $S V$ \\
\hline & & & Arequipa, Peru & 12.0 & $S V$ \\
\hline & & & LaPaz, Bolivia & 10.2 & $S V$ \\
\hline & & & LaPlata, Argentina & 10.2 & $S V$ \\
\hline
\end{tabular}

used by Asada and Takano (1963) to study the attenuation of compressional waves in the mantle. The Carnegie group (Steinhart et al., 1964) studied the spectrums of the phases $S c S$ and $S K P$ for a deep focus shock and concluded that a maximum value for $Q$ in the upper $600 \mathrm{~km}$ of the mantle was 160 and a minimum value for $Q$ in the lower mantle was 500 .

We have utilized information from shear waves reflected up to three times from the earth's core at near-normal incidence. From power spectrum analyses of the multiple $S c S$ and $s S c S$ phases from two deep focus South American shocks we have determined the average dissipation coefficient in the mantle for shear waves over the period range 14 to 70 seconds, and have estimated the dissipation in the regions of the mantle above and below the earthquake focus. A reasonable allowance for the variation of $Q$ with depth allows a conservative estimate to be made for $Q$ at the base of the mantle. A preliminary analysis of a portion of these data ap peared earlier (Anderson and Kovach, 1964).

\section{Analysis of Selected Earthquakes}

Table 1 lists the earthquakes and recording stations used. Figure 1 shows the long-period east-west seismogram of December 8, 1962, from Arequipa, Peru, showing five successive reflections of shear waves from the core at near normal 
incidence. The shock of December 8, 1962, had a focal depth of $620 \mathrm{~km}$ assigned by the USCGS but examination of the time intervals between multiple $s S c S$ and $S c S$ phases places the focal depth between 590 and $600 \mathrm{~km}$, depending on the earth model assumed. The earthquake of September 29, 1962, also has a focal depth of $600 \mathrm{~km}$ rather than the assigned depth of $575 \mathrm{~km}$.

Power spectra of the multiple $S c S$ and $s S c S$ phases recorded at Antofagasta, Chile, and Arequipa, Peru, for the shock of December 8, 1962, and La Paz, Bolivia, for the shock of September 29, 1962, were computed and are shown in figures 2, 3 and 4. Amplitude ratios were also measured from the seismograms at La Plata, Argentina, and La Paz, Bolivia, for the December 8 shock and agree with the computed spectral ratios.

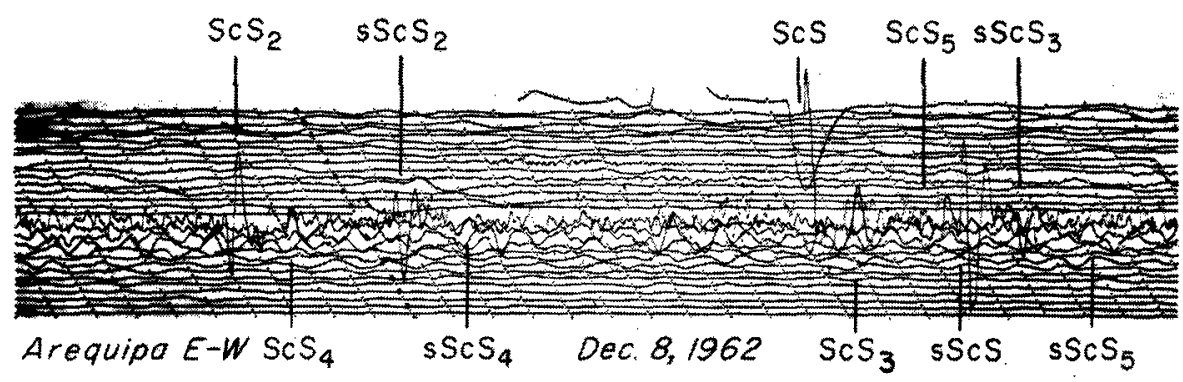

Frg. 1. Long-period east-west seismogram of December 8, 1962, Arequipa, Peru, showing five successive reflections from the core.

\section{Average Attenuation in the Mantle}

Amplitudes of body waves decrease during propagation due to a number of factors: geometrical spreading, effects of reflection and refraction at boundaries, scattering and absorption due to non-elastic processes.

For a particular ray path the geometric spreading can be computed from the expression (see Bullen, 1953, p. 124)

$$
\sqrt{\frac{\sin \theta}{r^{2} \sin \Delta \cos \theta_{0}}\left|\frac{d \theta}{d \Delta}\right|}
$$

where

$r=$ radius of earth

$\Delta=$ epicentral distance, constant for a single station experiment

$\theta_{0}=$ angle of emergence of ray at the earth's surface

$\theta=$ angle that ray leaves the source

This expression for geometric spreading was evaluated for the multiple $S c S$ phases using ray parameters appropriate to the CIT 11 standard earth model (Anderson and Toksöz, 1963). Figure 5 shows the spreading factor for a focal depth of $600 \mathrm{~km}$ for the multiple $S c S$ and $s S c S$ phases plotted against epicentral distance. Succes- 
sive reflections at the core and free surface boundary cause a focusing effect of the $s S c S$ ray relative to the corresponding $S c S$ ray. For example, even though $s S c S_{3}$ has travelled $1200 \mathrm{~km}$ more through the mantle relative to $S c S_{3}$ the correction for geometric spreading is almost the same.

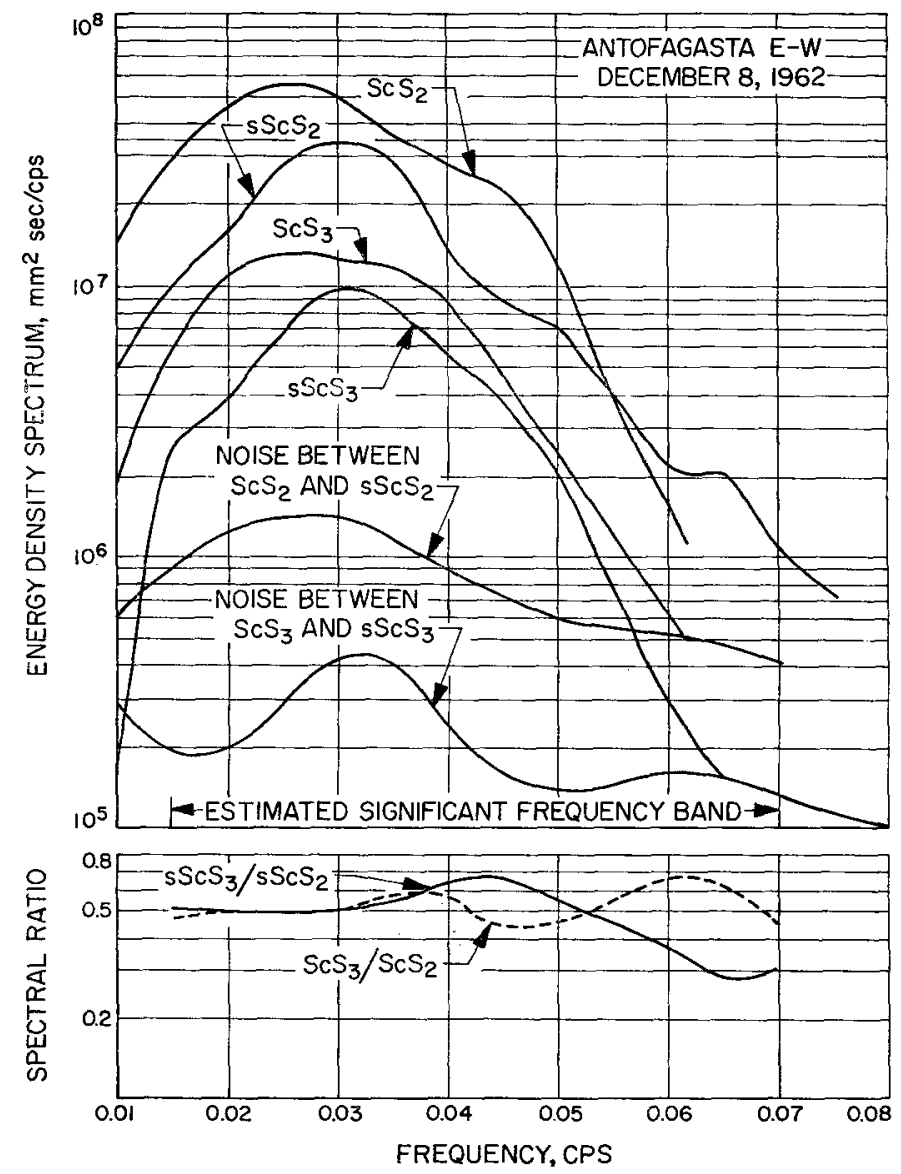

FIG. 2. Power spectra of multiple ScS phases for shock of December 8, 1962, recorded at Antofagasta, Chile.

For normal incidence the ratio of $S c S_{n+1} / S c S_{n}$, where $n$ is the number of reflections at the core can be written as

$$
\frac{S c S_{n+1}}{S c S_{n}}=R \bar{R} \exp \left[-2 k_{1} h_{1}\right] \exp \left[-2 k_{2} h_{2}\right] \frac{F_{n+1}}{F_{n}}
$$

where

$$
\begin{aligned}
& R=\text { reflection coefficient at core } \\
& \bar{R}=\text { reflection coefficient at surface }
\end{aligned}
$$


$h_{1}=$ focal depth

$h_{2}=H-h_{1}$

$H=$ depth of core

$k_{1}=$ average attenuation coefficient above focus

$k_{2}=$ average attenuation coefficient below focus

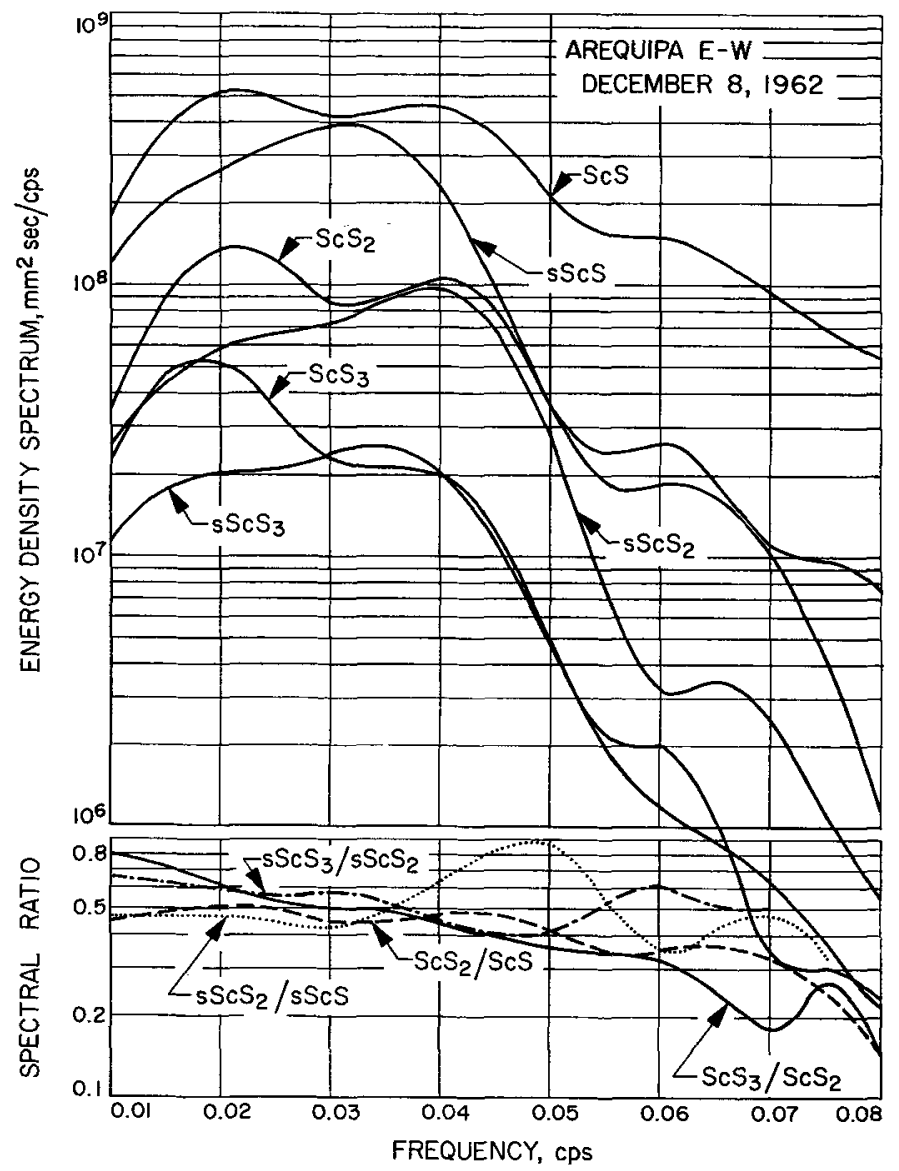

FIG. 3. Power spectra of multiple $S c S$ phases for shock of December 8, 1962, recorded at Arequipa, Peru.

$F_{n}=$ geometrie spreading factor for $S c S_{n}$

$f_{n}=$ geometric spreading factor for $s S c S_{n}$

Computed spectral ratios of $S c S_{n+1} / S c S_{n}$ or $s S c S_{n+1} / s S c S_{n}$, therefore, contain only information on the effects of reflection at the core boundary and free surface and transmission effects through the mantle.

The spectral amplitude ratios shown in figures 2,3 , and 4 show a general tendency to decrease with frequency. Irregularities in the individual spectral ratios are present but no other consistent feature is evident. If a transition or velocity gradient zone 
were present at the mantle-core boundary the reflection coefficient would be dependent on the frequency of the incident wave. The gross trend of the spectral amplitude ratios does not indicate a transition zone, at least on the scale of the wavelengths considered here.

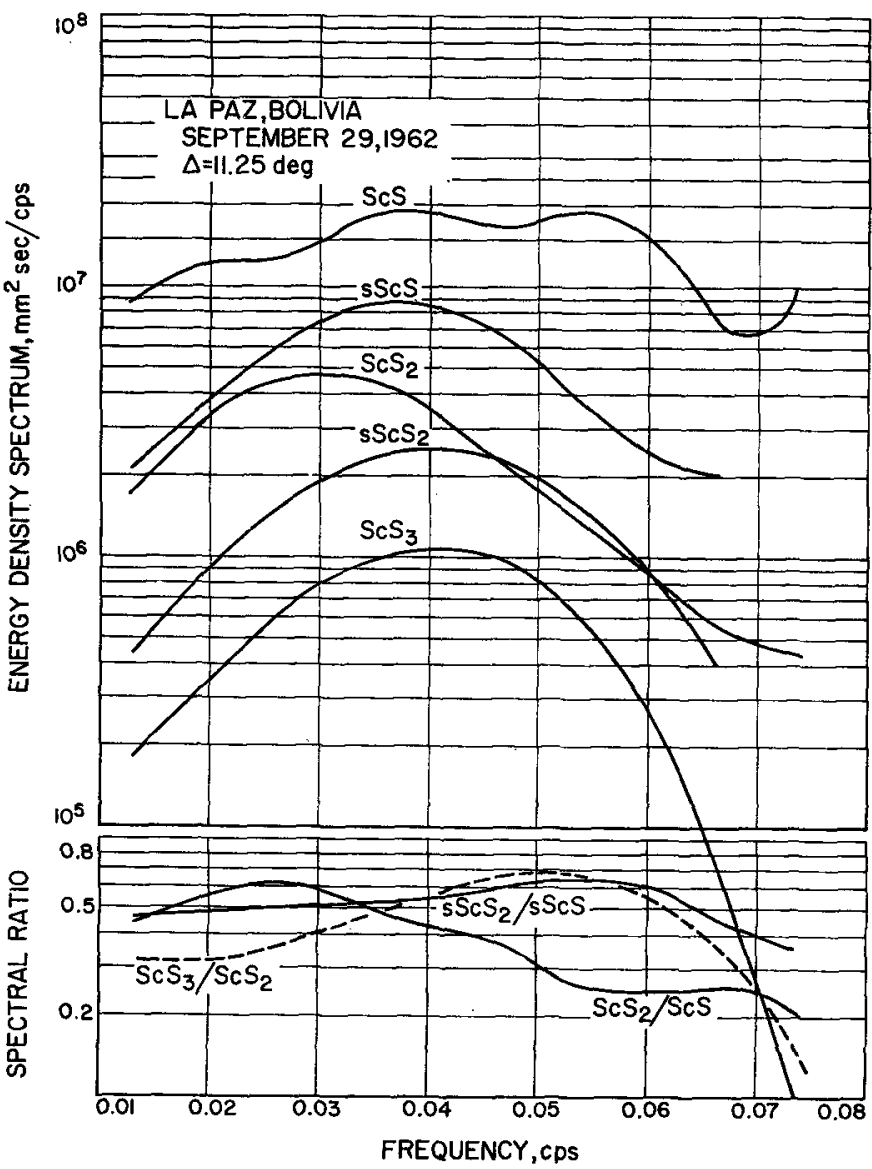

Frg. 4. Power-spectra of multiple $S c S$ phases for shock of September 29, 1962, recorded at La Paz, Boljvia.

The influence of a finite viscosity of the earth's core on the reflection of shear waves from the core boundary has been examined by Nakamura (1956) and Zharkov and Kalinin (1962). In examining the reflection of $S V$ waves from the core at near normal incidence Zharkov and Kalinin showed that the reflection coefficient for a solid-liquid interface is appropriate. For an incident $S H$ wave the presence of viscosity can give a reduction in amplitude and a change in phase of the reflected wave but a reasonable allowance for viscosity does not substantially alter the solidliquid reflection coefficient for near-normal incidence. Since the reflection coefficient for a solid-liquid interface is appropriate for $S H$ or $S V$ waves incident at the coremantle boundary $R$ may be set equal to unity. $\bar{R}$ may also be set equal to unity since total reflection occurs at a free surface for normally incident shear waves. 
Therefore, we may determine the average attenuation in the mantle from equation 2 by setting $k_{1}=k_{2}=k$

$$
\begin{aligned}
& k=-\log _{e}\left(\frac{S c S_{n+1}}{S c S_{n}} \cdot \frac{F_{n}}{F_{n+1}}\right) / 2 H \\
& Q=\pi f / \beta k
\end{aligned}
$$

The observed ratios were corrected for geometric spreading and averaged (figure 6 ). The standard deviation of the averaged ratios at specific frequencies is shown by the vertical lines. Using an average shear velocity in the mantle of $6.18 \mathrm{~km} / \mathrm{sec}$ determined from the interval times of the multiple $\operatorname{ScS}$ phases, lines of constant $Q$

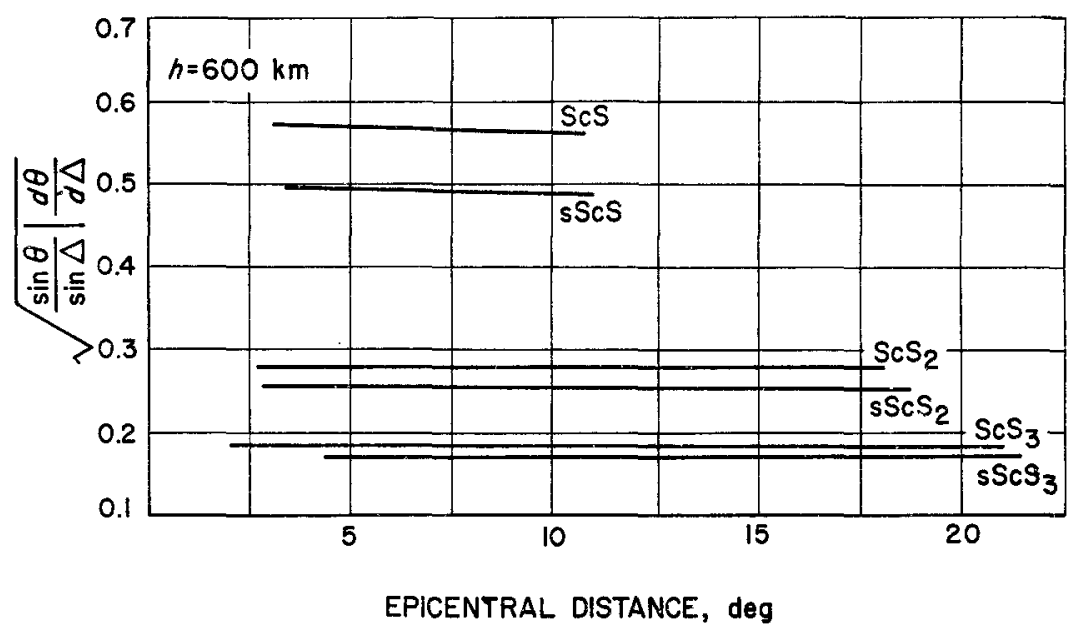

FIG. 5. Geometric spreading factor for a focal depth of $600 \mathrm{~km}$ for the multiple $S c S$ phases versus epicentral distance.

were computed and are also shown in figure 6 . Because of the fairly large scatter it cannot be definitely concluded that $Q$ for shear is independent of frequency over the range .015 to $.07 \mathrm{cps}$, but a $Q$ dependence on frequency is not required to satisfy the data. An average $Q$ of 600 for the mantle is compatible with the observations.

\section{Atrtenuation in the Upper and Lower Mantle}

We may determine the average absorption coefficient above the earthquake focus from the spectral ratios of $s S c S_{n} / S c S_{n}$ provided the same amount of energy is radiated upward as downward. Only an extended source having dimensions comparable to a wavelength and progressing unilaterally with a vertical component of velocity comparable to the shear velocity at the focal depth would have a radiation pattern in disagreement with this assumption.

Figure 7 shows the spectral ratios of $s S c S_{n} / S c S_{n}$ for the shocks of September 29, 1962, and December 8, 1962. Compared to the ratios of $S c S_{n+1} / S c S_{n}$ these ratios indicate the effect of an extra round trip through the upper $600 \mathrm{~km}$ of the earth and/ or the effect of radiation asymmetry. The spectral ratios for the two shocks were averaged separately and are also shown in figure 7. For frequencies greater than 
$0.03 \mathrm{cps}$ the averaged amplitude ratios show a general tendency to decrease with frequency. A linear decrease with frequency is the characteristic of a constant $Q$ material. An average $Q$ of 200 for shear waves in the upper $600 \mathrm{~km}$ of the mantle satisfies the data in the frequency range 0.03 to $0.07 \mathrm{cps}$. The assumption of radiation symmetry and the comments in the following paragraph make this an overestimate. An average shear velocity of $4.82 \mathrm{~km} / \mathrm{sec}$ was used for the upper $600 \mathrm{~km}$ of the mantle to compute $Q$.

For frequencies less than $0.03 \mathrm{cps}$ (longer periods) the spectral ratios decrease with increasing period. This behavior must be due to properties of the source or

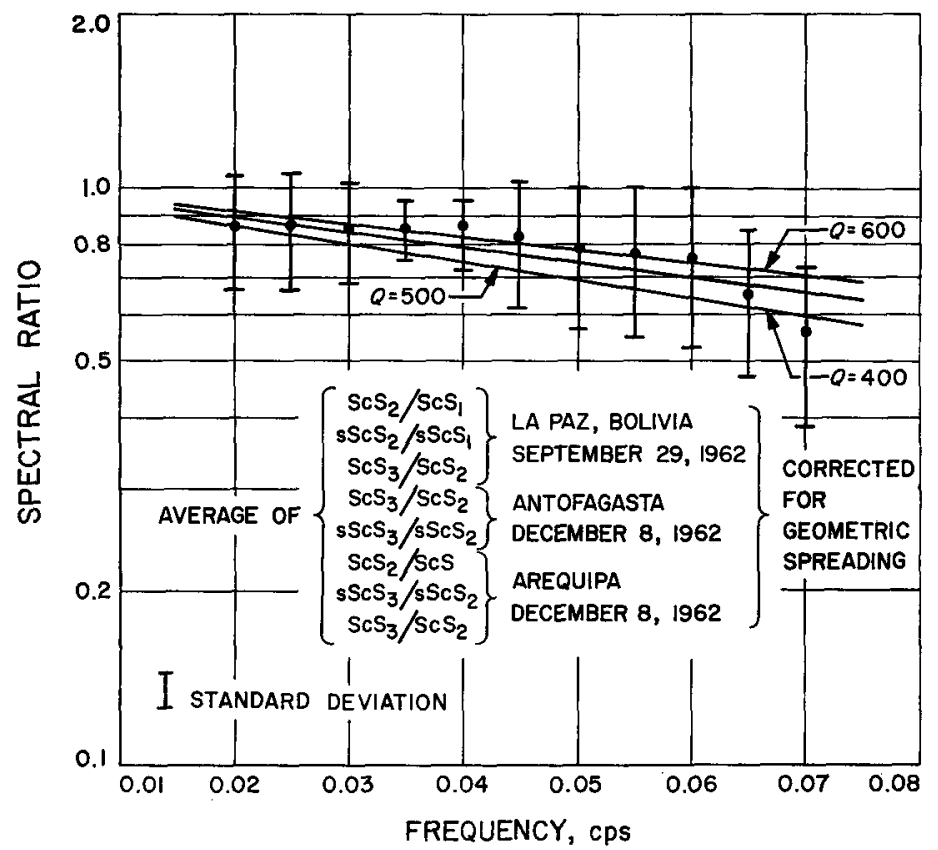

FIG. 6. Spectral ratios of $S c_{n+1} / S c S_{n}$ for the shocks of September 29, 1962, and December 8, 1962 .

properties of the upper mantle. Both earthquakes show this decrease for frequencies less than $0.03 \mathrm{cps}$. If this behavior is attributed to characteristics of the source this would imply that both deep focus shocks had similar source mechanisms. In this case, the source must be one in which the radiation pattern is asymmetric and frequency dependent; for example, a vertically travelling fault or a vertically growing phase change. Corrected spectral ratios of unity or greater must be due to properties of the source rather than properties of the upper mantle. The averaged spectral ratios for the shock of December 8, 1962, at a frequency of about $0.03 \mathrm{cps}$ are about unity and suggest a stress release zone growing vertically, although the data are not conclusive. The reduced amplitudes of $s S c S_{n} / S c S_{n}$ for frequencies less than $0.03 \mathrm{cps}$ can also be the effect of layering in the upper mantle having dimensions of hundreds of kilometers.

The average $Q$ for shear of 600 for the whole mantle and the estimate of 200 for $Q$ 


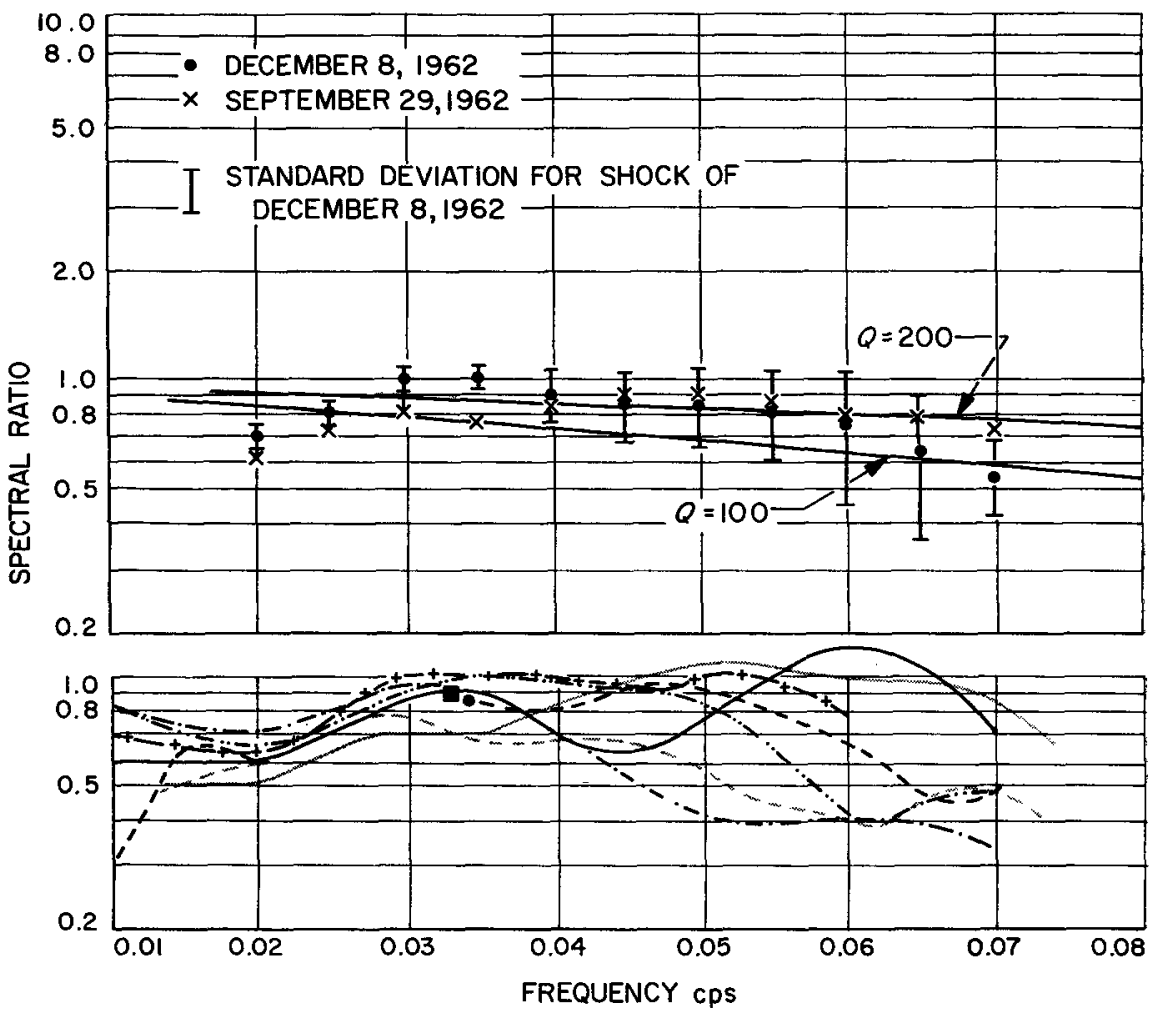

\begin{tabular}{|c|c|c|}
\hline \multicolumn{3}{|c|}{ DECEMBER 8,1962 } \\
\hline & $\mathrm{sScS}_{3} / \mathrm{ScS}_{3}$ LA PLATA, ARGENTINA & $\Delta=10.2 \mathrm{deg}$ \\
\hline & $\mathrm{sScS}_{2} / \mathrm{ScS}_{2}$ LA PLATA,ARGENTINA & $\Delta=10.2 \mathrm{deg}$ \\
\hline-- & $\operatorname{sScS}_{3} / \mathrm{ScS}_{3}$ ANTOFAGASTA, CHILE & $\Delta=6.7 \mathrm{deg}$ \\
\hline- & $\mathrm{sScS}_{2} / \mathrm{ScS}_{2}$ ANTOFAGASTA, CHILE & $\Delta=6.7 \mathrm{deg}$ \\
\hline -.-- & sScS / ScS AREQUIPA, PERU & $\Delta=10.2 \mathrm{deg}$ \\
\hline$-\cdot-$ & $\mathrm{sScS}_{2} / \mathrm{ScS}_{2}$ AREQUIPA, PERU & $\Delta=10.2 \mathrm{deg}$ \\
\hline+-+ & $\mathrm{sScS}_{3} / \mathrm{ScS}_{3}$ AREQUIPA, PERU & $\Delta=10.2 \mathrm{deg}$ \\
\hline & SEPTEMBER 29,1962 & \\
\hline$m$ & sScS/ScS LA PAZ, BOLIVIA & $A=11.30 \mathrm{deg}$ \\
\hline- & $\mathrm{SCS}_{2} / \mathrm{SCS}_{2} \angle A P A z$, DOLIUA & $s=1,3 \mathrm{deg}$ \\
\hline
\end{tabular}

FIG. 7. Spectral ratios of $s S c S n / S c S n$ for the shocks of September 29, 1962, and December 8, 1962.

for the upper $600 \mathrm{~km}$ of the mantle yield a value of at least 2200 for the average $Q$ of the lower mantle. This is an order of magnitude greater than the average $Q$ in the upper $600 \mathrm{~km}$ and over 3 times the average $Q$ of the whole mantle.

Anderson and Archambeau (1964) have developed a technique for determining $Q$ as a function of depth from the amplitude decay versus period of free oscillation and long-period surface wave data. The range of $Q$ for shear in the models which satisfy the available data is from about 80 in the upper mantle to about 2000 in 
the middle mantle. A rapid increase in $Q$ beginning at about $400 \mathrm{~km}$ seems to be required to satisfy the surface wave data. The average $Q$ 's for the upper and lower mantle determined here are in good agreement with the variation of $Q$ with depth indicated by the surface wave analyses. All of the above observations are consistent with an increase of $Q$ with depth in the lower mantle. We may use the average $Q$ of the lower mantle from the body wave data and starting values for $Q$ at $600 \mathrm{~km}$ depth compatible with the surface wave data to estimate the value for $Q$ at the base of the mantle. If we assume that the variation of $Q$ with depth in the lower mantle is governed primarily by pressure (Anderson and Archambeau, 1964, eqn. 31), then a value of 5000 is obtained as a conservative lower bound for $Q$ at the base of the mantle.

\section{ACKNowledgments}

This research was supported by Contract AF-49(638)-1337 of the Air Force Office of Scientific Research as part of the Advanced Research Projects Agency project VELA. The authors profited from conversations with Charles B. Archambeau, S. W. Smith and Frank Press. Computer programs written by Bruce Julian were used in this study.

\section{REFERENCES}

Anderson, D. L., and C. B. Archambeau

1964. "The Anelasticity of the Earth," J. Geophys. Res., 69: 2071-2084.

Anderson, D. L., and R. L. Kovach

1964. "Attenuation in the Mantle and Rigidity of the Core from Multiply Reflected Core Phases," Proc. Nat. Acad. Sci. U. S., 51: 168-172.

Anderson, D. L., and M. N. Toksöz

1963. "Surface Waves on a Spherical Earth, 1. Upper Mantle Structure from Love Waves," J. Geophys. Res., 68: 3483-3500.

Asada, T., and K. Takano

1963. "Attenuation of Short Period $P$ waves in the Mantle," $J$. Physics of the Earth, 11: $25-34$.

Bullen, K. E.

1953. An Introduction to the Theory of Seismology, (Cambridge University Press), 124.

Gutenberg, B.

1958. "Attenuation of Seismic Waves in the Earth's Mantle," Bull. Seism. Soc. Amer., 48: $269-282$.

Nakamura, $\mathbf{K}$.

1956. "On the Viscoelastic Nature of the Earth's Core," Sci. Reports Tohoku University Geophysics, 8: 93-110.

Press, F.

1956. "Rigidity of the Earth's Core," Science, 124: 1204.

Otsuka, M.

1963. "Some Considerations on the Wave Forms of ScS Phases," in Geophysical Papers Dedicated to Professor Kenzo Sassa, Kyoto, Japan.

Steinhart et al.

1964. Carnegie Institute of Washington Yearbook 62, 286-288.

Zharkov, V. N., and V. A. Kalinin

1962. "Seismic Wave Reflection at the Core-Mantle Boundary," Bull. (Izvestiya) Acad. of Sci., USSR (Geophys. Ser.), 299-302.

Division of Geological Sciences

California Institute of Technology

Pasadena, California

Contribution No. 1274

Manuseript received June 29, 1964. 